\title{
Dominique Pestre, À contre-science. Politiques et savoirs des sociétés contemporaines
}

Paris, Seuil, 2013

\section{Grégory Dufaud}

\section{(2) OpenEdition \\ Journals}

\author{
Édition électronique \\ URL : https://journals.openedition.org/artefact/8688 \\ DOI : $10.4000 /$ artefact. 8688 \\ ISSN : 2606-9245
}

Éditeur :

Association Artefact. Techniques histoire et sciences humaines, Presses universitaires du Midi

Édition imprimée

Date de publication : 11 septembre 2014

Pagination : 204-205

ISBN : 978-2-271-08150-6

ISSN : 2273-0753

Référence électronique

Grégory Dufaud, «Dominique Pestre, À contre-science. Politiques et savoirs des sociétés

contemporaines », Artefact [En ligne], 2 | 2014, mis en ligne le 11 mai 2021, consulté le 24 août 2021.

URL : http://journals.openedition.org/artefact/8688; DOI : https://doi.org/10.4000/artefact.8688

Ce document a été généré automatiquement le 24 août 2021.

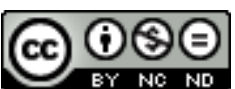

Artefact, Techniques, histoire et sciences humaines est mise à disposition selon les termes de la Licence Creative Commons Attribution - Pas d'Utilisation Commerciale - Pas de Modification 4.0 International. 


\section{Dominique Pestre, À contre-science. Politiques et savoirs des sociétés contemporaines}

Paris, Seuil, 2013

\section{Grégory Dufaud}

\section{RÉFÉRENCE}

Dominique Pestre, À contre-science. Politiques et savoirs des sociétés contemporaines, Paris, Seuil, 2013, 251 p.

1 Ce livre décrit à la fois le fonctionnement de la science et la relation réciproque qu'elle entretient avec la société. Le refus du dualisme, postulant l'existence de deux sphères distinctes, ouvre à une analyse complexe qui est cependant présentée de façon synthétique, claire et vivante. La démonstration s'appuie sur de nombreux exemples historiques et sociologiques. Si le propos de Dominique Pestre se veut méthodologique et épistémologique, il est également politique. Puisque les savoirs contribuent à la construction du monde social, il revient en effet aux scientifiques d'être vigilants quant à la portée des outils et des concepts qui sont les leurs. Aussi leur effort de réflexivité doit-il ne pas oublier d'examiner la dimension normative des énoncés qu'ils produisent. Cette invitation, formulée dans la troisième et dernière partie de l'ouvrage, s'appuie notamment sur une lecture de l'évolution des Sciences Studies depuis trente ans et des débats qu'elles ont connus : du programme de David Bloor et Harry Collins à celui de Bruno Latour et Michel Callon. D. Pestre plaide alors en faveur d'une analyse de la production scientifique qui rende toute la complexité du monde social, dans son hétérogénéité et sa violence, sans que soit privilégiée une échelle sur une autre (chap. 8). L'intérêt du livre réside justement dans la capacité de l'auteur à garder noués ensemble les trois fils de sa réflexion (méthodologique, épistémologique et politique). 
L'articulation entre les chapitres n'est toutefois pas toujours évidente : elle aurait sans doute mérité d'être plus explicite.

2 La première partie traite des "logiques scientifiques». D. Pestre montre que l'acte expérimental, s'il appartient à plusieurs mondes sociaux et physiques, procède également d'un «faire et d'un savoir faire» particuliers: le succès des expérimentations de James Joule est ainsi lié à son métier de brasseur (chap.2). Un résultat scientifique ne constitue toutefois pas une preuve en lui-même. Si une preuve est inscrite dans une situation, elle nécessite aussi un travail de conviction qui est toujours susceptible d'être remis en cause (chap. 1). Le cas de la découverte des ondes magnétiques à la fin du XIX ${ }^{e}$ siècle par Heinrich Hertz révèle non seulement la difficulté à obtenir un résultat expérimental solide, mais aussi l'importance du jugement du scientifique dans la décision de le diffuser ou non. Les travaux de Louis Néel sur la protection des navires contre les mines magnétiques allemandes pendant la « drôle de guerre » montrent, quant à eux, que les critères de persuasion diffèrent en fonction des acteurs et des opérations de cadrage liées à leur activité. La science produit donc un savoir qui tient à des pratiques concrètes. Mais, parce que ses démarches sont par définition limitatives, elles génèrent également, et en même temps, de l'ignorance (chap.3). C'est une de ses limites que les "marchands de doute" cherchent à instrumentaliser à des fins politique et économique. Aussi D. Pestre appelle-t-il à l'élaboration de politiques de la recherche qui assument davantage cette limite. Les propositions qu'il formule en ce sens visent à placer les scientifiques au sein des débats publics.

3 La deuxième partie du livre évoque les « logiques économiques, sociales et politiques » des sciences. Les trente dernières années sont en effet marquées par une série de transformations (chap.4). C'est tout d'abord l'économie politique des savoirs qui a évolué : accroissement des acteurs de la recherche, redistribution géographique de l'innovation, extension de la conception de propriété intellectuelle et réduction de l'autonomie des universitaires. Les pratiques et les disciplines scientifiques ont aussi changé : développement de la technobiologie, recours aux outils mathématiques et informatiques, retour des sciences de l'observation et essor des sciences du système Terre. Le savoir et la science connaissent des modalités nouvelles de contrôle avec la montée de la participation citoyenne (chap.6). Lorsqu'elle est spontanée, la participation citoyenne ne joue un rôle régulateur qu'après coup, dans la mesure où elle concerne un produit technique et scientifique déjà mis en circulation. Sous sa forme instituée, et sauf en de rares cas où elle ne peut être évitée, les logiques du marché, pour qui elle représente un frein, tendent à la marginaliser pour lui préférer des petits comités plus souples et plus dociles.

D'un point de vue social, le fait majeur a été l'érosion de l'idée de progrès en raison des controverses autour du nucléaire et de la montée de la cause environnementale. C'est la notion de "risque » qui est devenue centrale dans nos sociétés contemporaines (chap. 5). En France, son origine remonte au lendemain de la Révolution et à l'ordre économique qui s'installe alors : elle renvoie au souci de prévisibilité des entrepreneurs qui cherchaient à garantir leurs investissements. Les comités, créés pour apprécier les dangers des nouveaux produits mis sur le marché, fonctionnaient toutefois dans le premier $\mathrm{xx}^{\mathrm{e}}$ siècle comme des instances visant à faire accepter les effets négatifs du développement capitaliste. Après la Seconde Guerre mondiale, l'idée de risque est devenue omniprésente. Elle participe de la reformulation des enjeux liés à l'équité 
sociale et aux logiques de gouvernement. Une autre notion aujourd'hui importante est celle de " développement durable » qui cherche à définir un équilibre entre croissance économique, justice sociale et gestion des ressources naturelles (chap. 7). Cet idéal se heurte dans les faits à des modes de régulation sur lesquels il n'a pas prise. Il n'en est pas moins à l'origine d'actions et de pratiques dont les résultats restent néanmoins limités. La vision du monde qu'il symbolise explique les violentes attaques qu'il doit affronter en provenance des milieux néolibéraux et conservateurs américains.

Pour conclure, si D. Pestre aspire à davantage de modestie de la part de la science, en ce qu'elle accepterait les limites et les points aveugles qui sont les siens, c'est donc pour mieux (re)poser la question des choix démocratiques au regard des évolutions rencontrées par les sociétés contemporaines depuis une trentaine d'années. On espère que son appel sera entendu !

\section{AUTEURS}

GRÉGORY DUFAUD

LabEx tepsis, Cercec (EHESS) 